\title{
Effect of soliton and vortex geometry on the transport of Bose-Einstein condensates in optical lattices
}

\author{
R. G. Scott, ${ }^{1}$ A. M. Martin, ${ }^{1,2}$ and T. M. Fromhold ${ }^{1}$ \\ ${ }^{1}$ School of Physics and Astronomy, University of Nottingham, Nottingham NG7 2RD, United Kingdom \\ ${ }^{2}$ School of Physics, University of Melbourne, Victoria 3010, Australia
}

(Received 2 February 2004; published 11 June 2004)

\begin{abstract}
We investigate the effect of solitons and vortices, with a range of different topologies, on the dynamics of Bose-Einstein condensates in a one-dimensional optical lattice and a three-dimensional harmonic trap. The solitons and vortices are imposed on the initial state of the atom cloud, which is set in oscillatory motion by a sudden displacement of the harmonic trap. We study this motion using both quantum-mechanical calculations, based on the nonlinear Schrödinger equation, and a semiclassical model for transport through the lowest energy band of the optical lattice. We demonstrate that the geometry of the vortices and solitons in the initial state plays a crucial role in the evolution of the atom cloud. In particular, we find that the center-of-mass motion of a condensate containing a vortex is only weakly damped if the vortex core lies parallel to the optical lattice direction, but is strongly damped when the core is orientated perpendicular to the lattice direction. Severe damping is observed for a condensate containing a soliton whose nodal plane is perpendicular to the optical lattice.
\end{abstract}

DOI: 10.1103/PhysRevA.69.063607

PACS number(s): 03.75.Kk, 05.45.Yv, 32.80.Pj, 42.50.Vk

\section{INTRODUCTION}

Recent studies of Bose-Einstein condensates (BECs) in optical lattices have been a rich source of physics, broadening the current understanding of energy-band transport [1-7], condensate excitations and instabilities [3,5,6,8-14], and the role of interatomic interactions $[3,4,7,10,15]$. By accelerating the condensate along the optical lattice direction, experimentalists have investigated the quantum transport of BECs in periodic potentials, and successfully observed Bloch oscillations $[1,2,10,15]$. These experiments stimulated considerable theoretical interest $[3-5,10,15-18]$, which focused on damping and disruption of the Bloch oscillations and, in particular, how the dynamics of the BEC are affected by the interatomic interactions, described by the nonlinear term in the GrossPitaevskii equation. Several different mechanisms have been proposed for the breakdown of Bloch motion, including the screening of the optical potential [4], Landau Zener tunneling $[1,2,4,10,17]$, looped Bloch bands [17], dynamical instability [10], and the production of solitons and vortices [18].

Previous studies of the motion of condensates in optical lattices have considered the acceleration of the condensate ground state [3-5,15-18]. However, more complicated condensate wave functions containing topological excitations such as solitons and vortices have been realized experimentally $[11,13,14,19-24]$ and simulated theoretically [11-14,25-27]. Solitons may be generated by phase imprinting and/or density engineering [11-14,20,21], and by magnetic tuning of the interatomic interactions [19]. Vortices have been created from the decay of solitons [11,21], by phase engineering $[20,27]$, stirring with a focused laser beam $[22,23]$, or by moving a laser beam through the atom cloud above a critical velocity $[24,26]$. Studies of condensates that have been excited in these ways have examined the stability and energy of the excitations [12,21,25], their dynamics within the atom cloud $[14,28]$, and interactions between excitations [12].
In previous work [18,29], we considered the motion of ground-state condensates that are accelerated through an optical lattice. We showed that Bragg reflection can generate solitons, which decay into vortex rings via the snake instability $[11,21]$, thereby damping the center-of-mass motion and, for certain densities and accelerations, causing a dramatic explosion of the atom cloud. In this paper, we examine how excited condensates move through optical lattices, by considering wave functions that initially contain a soliton or a vortex. Our quantum-mechanical ${ }^{1}$ and semiclassical calculations demonstrate that these initial excitations have a pronounced effect on the evolution of the atom cloud and on the damping of the center-of-mass motion. In particular, we show that the geometry and orientation of the initial excitation is crucial for determining the damping rate and the longterm behavior of the atom cloud.

We consider three condensates with different initial excitations, which we refer to as condensates $A, B$, and $C$. Condensate $A$ initially contains one soliton, whose nodal plane lies perpendicular to the axis of the optical lattice. Condensate $B$ initially contains a single vortex line, whose core (axis of rotation) is orientated along the optical lattice direction. Condensate $C$ also initially contains a single vortex line, but its core lies perpendicular to the optical lattice direction. As in recent experiments [8], we accelerate the condensates by abruptly displacing the harmonic trap along the optical lattice axis. In response, the condensates all perform damped Bloch oscillations. However, the extent of the damping is

\footnotetext{
${ }^{1}$ Our "quantum-mechanical" calculations are, in fact, solutions of the Gross-Pitaevski equation. This equation is, strictly speaking, not a full quantum-mechanical treatment, but a nonlinear Schrödinger equation in which the effect of the interatomic interactions is approximated by a mean-field approach. For brevity, we refer to our solutions of the Gross-Pitaevskii equation as "quantum mechanical."
} 
different in each case. For condensate $B$, the damping originates from the production of solitons and vortices that evolve from the standing wave formed at the point of Bragg reflection [18]. However, the damping observed for condensates $A$ and $C$ is far more severe than for condensate $B$ because the initial topological excitations impede the formation of the standing wave at the point of Bragg reflection. As a consequence, the internal structure of the condensate is strongly disrupted, leading to the formation of further topological excitations and the expansion of the atom cloud.

In Sec. II we specify the geometry of the optical lattice and the harmonic trap, together with the generic properties of the three distinct condensates. Specific features of condensates $A, B$, and $C$ and their dynamics are considered in Secs. III, IV, and V, respectively.

\section{THEORETICAL MODEL OF THE BOSE-EINSTEIN CONDENSATE}

As in previous work $[18,29]$, we consider Bose-Einstein condensates formed from $N_{A}=10^{4}{ }^{87} \mathrm{Rb}$ atoms in a threedimensional harmonic trap and a one-dimensional optical lattice. The optical lattice is formed by two counter-propagating laser beams, each of wavelength $\lambda=795 \mathrm{~nm}$, as in recent experiments [8]. The potential energy of a single atom in the optical lattice is

$$
V_{\mathrm{OL}}(x)=V_{0} \sin ^{2}(\pi x / d),
$$

where $d=\lambda / 2=397.5 \mathrm{~nm}$ and $V_{0}=23.3 \mathrm{peV}(=1.55$ times the recoil energy $E_{R}=\hbar^{2} k^{2} / 2 m$, where $k$ is the wave vector of the laser light and $m$ is the mass of a single ${ }^{87} \mathrm{Rb}$ atom) are, respectively, the period and depth of the optical potential. Due to its translational symmetry, the optical lattice generates an energy-band structure for motion along the $x$ direction. We calculated the energy wave vector dispersion relations for the two lowest energy bands using Mathieu functions [30]. The lowest energy band of the optical lattice lies between 0.70 and $1.37 E_{R}$, and the bottom of the second band is at $4.84 E_{R}$, which is well above the top of the optical potential. In principle, the harmonic trap breaks the translational symmetry of the optical potential. However, for the parameters considered in this paper, the condensate remains sufficiently close to the trap center to ensure that the change in trap potential across each lattice period is much less than the width of the lowest energy band. Consequently, the trap potential can be treated as a perturbation to the optical lattice and the band structure remains intact $[18,29,31]$. Furthermore, there is a large energy gap between the first and second bands, so Landau-Zener tunneling into the second band is negligible.

The harmonic trap is spherically symmetrical. Its potential energy profile is given by

$$
V_{\mathrm{TRAP}}(x, y, z)=\frac{m \omega^{2}}{2}\left(x^{2}+y^{2}+z^{2}\right),
$$

where $\omega=2 \pi \times 50 \mathrm{rad} \mathrm{s}^{-1}$ is the trap frequency. The total potential energy of a single atom in the optical lattice and harmonic trap is

$$
V_{\mathrm{T}}(x, y, z)=V_{\mathrm{OL}}(x)+V_{\mathrm{TRAP}}(x, y, z) .
$$

We describe the evolution of the condensate wave function $\Psi$ by numerical integration of the time-dependent Gross-Pitaevskii equation, given by

$$
\begin{aligned}
i \hbar \frac{\partial \Psi(x, y, z, t)}{\partial t}= & {\left[-\frac{\hbar^{2}}{2 m} \nabla^{2}+V_{\mathrm{T}}(x, y, z)\right.} \\
& \left.+U_{0}|\Psi(x, y, z, t)|^{2}\right] \Psi(x, y, z, t),
\end{aligned}
$$

where $\nabla^{2}$ is the Laplacian and

$$
U_{0}=\frac{4 \pi \hbar^{2} a}{m},
$$

in which $a=5.4 \mathrm{~nm}$ is the $s$-wave scattering length [32]. The condensate wave function $\Psi$ is normalized so that $|\Psi|^{2}$ is the number of atoms per unit volume. In our calculations, we express Eq. (4) as a finite-difference equation, and then solve it numerically using the Crank-Nicolson method, incorporating time or operator splitting [33].

\section{DYNAMICS OF CONDENSATE $A$}

The initial wave function of condensate $A$ is chosen to contain one stationary soliton, whose nodal plane lies perpendicular to the axis of the optical lattice. Experimentally, this can be achieved by density and/or phase imprinting techniques [11-14,20,21]. The condensate has cylindrical symmetry, and is therefore conveniently described in cylindrical polar coordinates $(x, r, \varphi)$, where $r=\sqrt{y^{2}+z^{2}}$ and $\varphi$ $=\tan ^{-1}(y / z)$. Since neither the total potential $V_{\mathrm{T}}(x, r)$ nor the initial condensate wave function has any $\varphi$ dependence, the three-dimensional Gross-Pitaevskii equation (4) can be reduced to an effective two-dimensional equation $[18,29]$, given by

$i \hbar \frac{\partial \Psi(x, r, t)}{\partial t}=\left[-\frac{\hbar^{2}}{2 m} \nabla_{c}^{2}+V_{\mathrm{T}}(x, r)+U_{0}|\Psi(x, r, t)|^{2}\right] \Psi(x, r, t)$,

where the Laplacian in cylindrical polar coordinates is

$$
\nabla_{c}^{2}=\frac{\partial^{2}}{\partial x^{2}}+\frac{\partial^{2}}{\partial r^{2}}+\frac{1}{r} \frac{\partial}{\partial r} .
$$

We calculate the initial wave function using an imaginary time algorithm [34], and obtain the density profile shown in Fig. 1(a). Note that the soliton (broad light band) becomes wider at the edges of the condensate, where the atom density $n(x, r)$ is lower, and hence the local healing length $l(x, r)$ $=1 / \sqrt{8 \pi n(x, r) a}$ is longer.

At time $t=0$, we set the condensate in motion by abruptly displacing the harmonic trap by $25 \mu \mathrm{m}$ along the $x$ axis. This increases the atom cloud's potential energy, which is converted into kinetic energy as the condensate accelerates towards the trap potential minimum. We determined the equivalent single-particle trajectory using the semiclassical equations of motion $d x / d t=\hbar^{-1} d E\left(k_{x}\right) / d k_{x}$ and $d k_{x} / d t$ 


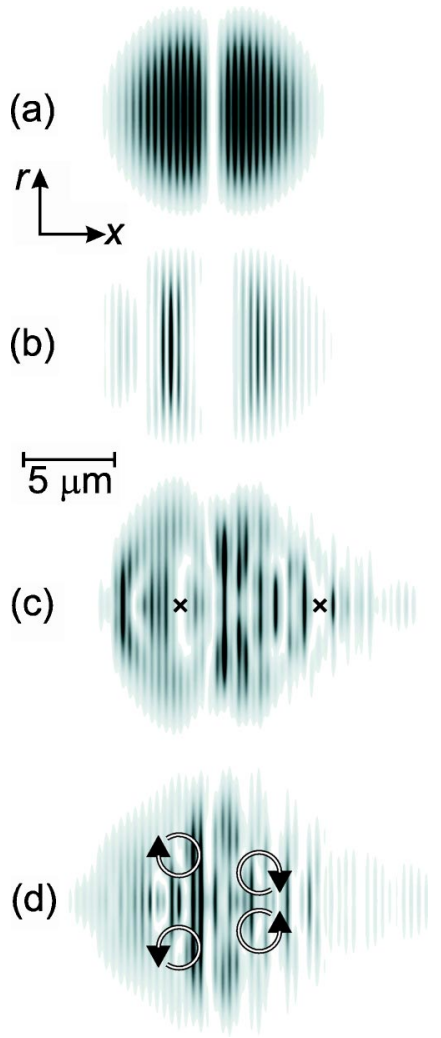

FIG. 1. Gray scale plots of density (white $=0$, black $=$ high) in the $x-r$ plane (axes inset) for condensate $A$ at $t=0 \mathrm{~ms}$ (a), $2.6 \mathrm{~ms}$ (b), $5 \mathrm{~ms}$ (c), and $6.1 \mathrm{~ms}$ (d). The crosses in (c) mark solitons produced by the first Bragg reflection. Arrows in (d) enclose vortices and show the direction of condensate rotation. The horizontal bar shows scale.

$=\hbar^{-1} F_{x}$ [35], where $E\left(k_{x}\right)$ is the energy wave-number dispersion relation for the lowest energy band, and $F_{x}=-m \omega^{2} x$ is the restoring force along the $x$ axis due to the harmonic trap. Solving these equations of motion yields the semiclassical $x(t)$ trajectory shown by the dashed curve in Fig. 2(a). This trajectory shows that, following the trap displacement, a pointlike particle performs regular Bloch oscillations in the lowest energy band of the optical lattice. The upper turning points in the $x(t)$ curve occur when the particle comes to rest at the top of the energy band (corresponding to the Bragg reflection of the matter waves), and the lower turning points are due to reflection bounded by the magnetic trap. The Bloch oscillations have a period of $5.1 \mathrm{~ms}$ and a peak-topeak amplitude of $5.0 \mu \mathrm{m}$.

We now compare the semiclassical trajectory with the corresponding quantum-mechanical mean $x$ position of condensate $A,\langle x\rangle$, which is shown as a function of $t$ by the solid curve in Fig. 2(a). The amplitude and period of the first Bloch oscillation are $4.7 \mu \mathrm{m}$ and $5.1 \mathrm{~ms}$, in good agreement with the corresponding semiclassical calculation for a pointlike particle. But the amplitude of subsequent oscillations decreases dramatically as $t$ increases. After three oscillations ( $t \approx 15 \mathrm{~ms}$ ), the motion almost completely breaks down, and the condensate's center-of-mass remains approximately stationary. The deviation of the quantum-mechanical $\langle x\rangle$ vs $t$ curve from the semiclassical trajectory, $x(t)$, indicates that

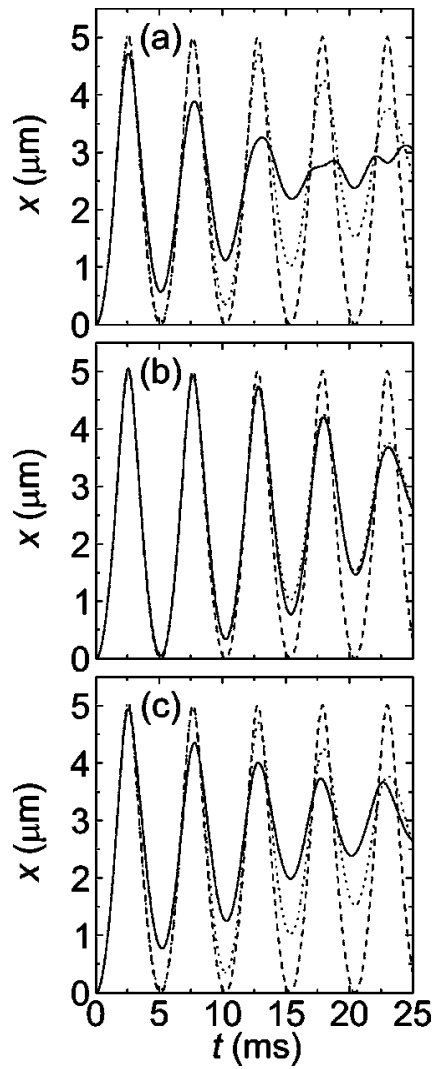

FIG. 2. Solid curves: $\langle x\rangle$ vs $t$ for condensate $A$ (a), condensate $B$ (b), and condensate $C(\mathrm{c})$. The dashed curve in all figures: equivalent semiclassical trajectory. The dotted curve in all figures: $\langle x\rangle$ vs $t$ for a condensate that is initially in its ground state, but otherwise identical to condensates $A-C$.

the condensate's center-of-mass motion is damped by processes that cannot be described in a semiclassical singleparticle model of energy-band transport.

The dotted curve in Fig. 2(a) shows $\langle x\rangle$ calculated as a function of $t$ for a condensate that is identical to condensate $A$, except that, initially, the atom cloud is in its ground state, and therefore contains no soliton. This curve shows that the condensate ground state also performs damped Bloch oscillations. However, the damping, which originates from soliton and vortex production (see Refs. [18,29] for a detailed explanation), is much less severe than that for condensate $A$. This demonstrates that the presence of the initial soliton in condensate $A$ strongly enhances the damping processes.

The damping mechanism can be understood by considering changes in the internal structure of the atom cloud, which is shown in Fig. 1 at various key stages of the evolution. As discussed in our previous work on BECs with no initial excitations [18,29], when a condensate undergoes Bragg reflection, a standing wave is formed, imprinting a density node and a $\pi$-phase shift in the condensate wave function at each maximum in the optical lattice potential. However, in condensate $A$, the presence of the soliton in the initial state disrupts the formation of the standing wave on Bragg reflection. To demonstrate this, we show the condensate density profile at the point of the first Bragg reflection in Fig. 1(b). Although the standing wave does form locally in some regions of the 
condensate, the atom cloud has become fragmented, with large areas of low atom density. This disruption allows the production of further solitons, two of which are marked by crosses in Fig. 1(c). These solitons then decay via the snake instability into vortex rings. Cross sections through two vortex rings are shown in Fig. 1(d), encircled by arrows indicating the direction of quantized circulation. This rapid production of topological excitations leads to further fragmentation and expansion of the atom cloud, and so causes a complete breakdown of the Bloch motion.

\section{DYNAMICS OF CONDENSATE $B$}

Condensate $B$ initially contains a single vortex line whose axis of rotation is orientated along the optical lattice $(x)$ direction. We take the associated $x$ component of each atom's angular momentum to be $\hbar$, so that in cylindrical polar coordinates, the condensate wave function is

$$
\Psi(x, r, \varphi, t)=\psi(x, r, t) e^{i \varphi} .
$$

By substituting this wave function into Eq. (4), expressed in cylindrical polar form, the three-dimensional time-dependent Gross-Pitaevskii equation can be reduced to an effective twodimensional equation, given by

$$
\begin{aligned}
i \hbar \frac{\partial \psi(x, r, t)}{\partial t}= & {\left[-\frac{\hbar^{2}}{2 m} \nabla_{c}^{2}+\frac{\hbar^{2}}{2 m r^{2}}+V_{\mathrm{T}}(x, r)\right.} \\
& \left.+U_{0}|\psi(x, r, t)|^{2}\right] \psi(x, r, t) .
\end{aligned}
$$

The initial wave function of the condensate is obtained by solving Eq. (9), using an imaginary time algorithm [34]. Figure 3(a) shows the density profile of this initial state. As for the soliton in condensate $A$, the vortex (indicated by the light band along the $x$ direction) becomes wider at the left- and right-hand edges of the condensate, where the atom density is lower and hence the local healing length is longer.

As for condensate $A$, at time $t=0$ we set the condensate in motion by abruptly displacing the harmonic trap by $25 \mu \mathrm{m}$ along the $x$ axis. The subsequent evolution of the condensate's quantum-mechanical mean position, $\langle x\rangle$, is shown by the solid curve in Fig. 2(b). The corresponding semiclassical trajectory is represented by the dashed curve in the same figure. As before, the condensate performs damped Bloch oscillations, but the damping is much less severe than for condensate $A$. The Bloch motion is still clear after five oscillations, although the amplitude of the oscillations has halved during the simulation. The rate of damping is very similar to that found in the $\langle x\rangle$ vs $t$ curve calculated for a condensate that is initially in its ground state, but otherwise equivalent to condensate $B$ [dotted line in Fig. 2(b)].

This behavior can be understood by studying the internal structure of condensate $B$, which is shown at various key stages of the simulation in Fig. 3. As discussed in the previous section, the standing wave formed at the point of each Bragg reflection imprints density nodes and $\pi$-phase shifts on the condensate wave function. But in contrast to condensate $A$, the standing wave forms on the first Bragg reflection (a)
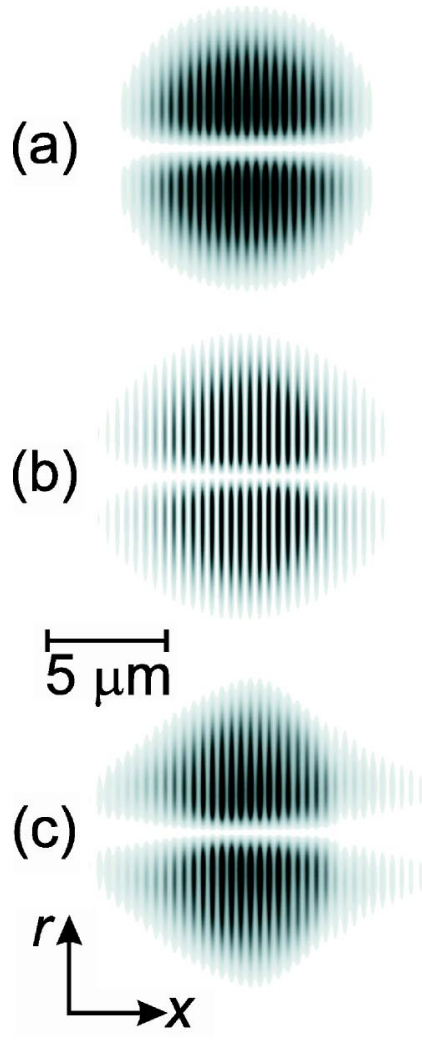

(d)

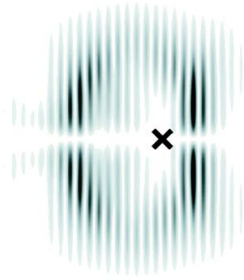

(e)

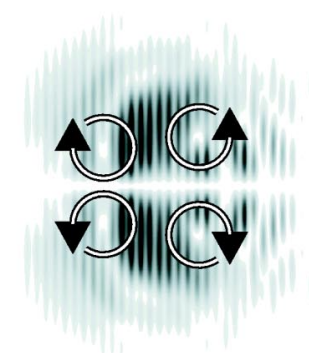

FIG. 3. Gray scale plots of density (white $=0$, black $=$ high) in the $x-r$ plane (axes inset) for condensate $B$ at $t=0 \mathrm{~ms}$ (a), $2.6 \mathrm{~ms}$ (b), $4 \mathrm{~ms}$ (c), $7.8 \mathrm{~ms} \mathrm{(d),} \mathrm{and} 11.3 \mathrm{~ms}$ (e). The cross in (d) marks a soliton produced by Bragg reflection. The arrows in (e) enclose vortices and show direction of condensate rotation. The horizontal bar shows scale.

without causing any disruption to the atom density profile, as shown in Fig. 3(b). No additional topological excitations are produced, and following Bragg reflection the condensate regains a density profile [Fig. 3(c)] that is very similar to that of the initial condensate wave function. There has been only a slight distortion and expansion of the atom cloud during the first oscillation. However, on the second Bragg reflection, the condensate has become sufficiently disrupted to allow dark solitons to form in response to the density nodes and 
$\pi$-phase shifts present in the standing wave. One of these solitons is marked by the cross in Fig. 3(d), which shows the condensate density profile at $t=7.8 \mathrm{~ms}$, approximately midway between the second and third Bragg reflections. The solitons then decay via the snake instability into vortex rings, two of which are encircled by arrows in Fig. 3(e), indicating the direction of circulation. It should be noted that these vortex rings have quantized circulation around the central vortex line, in addition to quantized circulation around their own cores. These structures are known as skyrmions [27].

The evolution of condensate $B$ is very similar to that reported in our previous paper [18], in which we considered a condensate that was initially in its ground state. Hence we conclude that a vortex whose axis of rotation is parallel to the optical lattice axis has little effect on the condensate dynamics. This is because the motion of the condensate about the vortex is perpendicular to the optical lattice axis, and so it does not interfere with the process of Bragg reflection.

\section{DYNAMICS OF CONDENSATE $C$}

Condensate $C$ initially contains a single vortex line whose axis of rotation lies along the $z$ direction, perpendicular to the optical lattice direction. It differs from condensates $A$ and $B$ because its atom density profile does not have cylindrical symmetry. Consequently, it is impossible to reduce the threedimensional problem to an equivalent effective twodimensional problem by symmetry considerations. However, we can achieve this reduction, which is required to facilitate finite difference solution of the Gross-Pitaevskii equation, by assuming that the condensate is much more strongly confined along the $z$ direction than along the $x$ and $y$ directions. In this case, we can write the Gross-Pitaevskii equation (4) in the two-dimensional form

$$
\begin{aligned}
i \hbar \frac{\partial \Psi(x, y, t)}{\partial t}= & {\left[-\frac{\hbar^{2}}{2 m}\left(\frac{\partial^{2}}{\partial x^{2}}+\frac{\partial^{2}}{\partial y^{2}}\right)+V_{\mathrm{T}}(x, y)\right.} \\
& \left.+U_{0}|\Psi(x, y, t)|^{2}\right] \Psi(x, y, t) .
\end{aligned}
$$

We find the initial condensate wave function using an imaginary time algorithm [34], subject to the requirement that there is a $2 \pi$-phase change in the condensate wave function around the trap center, which corresponds to a quantized angular momentum of $\hbar$. The wave function is normalized according to

$$
\int|\Psi(x, y, t)|^{2} d x d y=C=\frac{N_{A}}{L_{z}},
$$

where $L_{z}$ is the confinement length in the $z$ direction. We chose $C=1.7 \times 10^{9} \mathrm{~m}^{-1}$, so that the peak density of condensate $C$ is identical to that of condensate $B$, thereby ensuring that the two systems can be directly compared. The resulting initial state is shown in Fig. 4(a), in which the vortex is clearly visible at the center of the atom cloud.

As for condensates $A$ and $B$, the atom cloud is set in motion by abruptly displacing the harmonic trap by $25 \mu \mathrm{m}$ along the $x$ axis at time $t=0$. The subsequent evolution of the
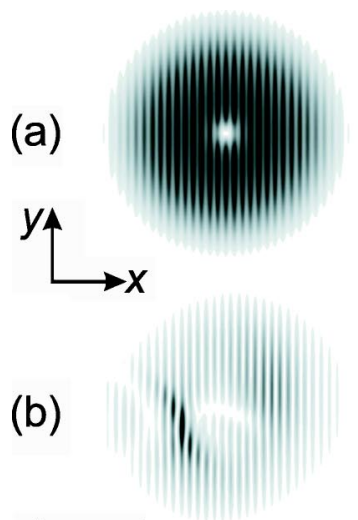

(c)

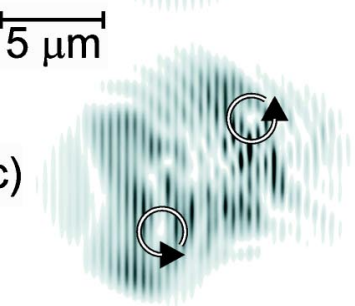

(d)

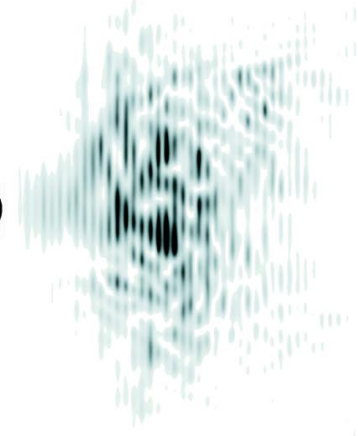

FIG. 4. Gray scale plots of density (white $=0$, black=high) in the $x-y$ plane (axes inset) for condensate $C$ at $t=0 \mathrm{~ms}$ (a), $2.6 \mathrm{~ms}$ (b), $4 \mathrm{~ms}$ (c), and $11.3 \mathrm{~ms}$ (d). The arrows in (c) enclose vortices and show the direction of condensate rotation. The horizontal bar shows scale.

condensate's quantum-mechanical mean position is shown by the solid line in Fig. 2(c). As before, the condensate performs damped Bloch oscillations, but the damping is more severe than for both condensate $B$ and a condensate that is initially in its ground state, which therefore contains no vortex [shown by the dotted curve in Fig. 2(c)]. This can be understood by considering the internal structure of the atom cloud, shown at various key stages of the simulation in Fig. 4. Figure 4(b) shows the condensate density profile at the point of the first Bragg reflection $(t=2.6 \mathrm{~ms})$. In contrast to the corresponding image for condensate $B$, shown in Fig. 3(b), the formation of the standing wave has disrupted the internal structure of the condensate, imposing spatial fluctuations in the atom density that extend over several lattice periods. Despite this disruption, the standing wave is locally intact in some regions of the condensate, giving the density profile a broadly similar character to the corresponding image for condensate $A$, shown in Fig. 1(b). Shortly after the first Bragg reflection, these changes in the atom density profile allow the formation of solitons and vortices, as shown in 
Fig. 4(c). Two of the vortices are encircled by arrows, which indicate the direction of circulation.

This behavior occurs because the circulation of the condensate about the initial vortex breaks the atom cloud's symmetry under reflection about the $x$ axis, and, as a consequence, disrupts the formation of the standing wave at the point of the first Bragg reflection. Hence, further topological excitations are produced, leading to an expansion of the atom cloud and a damping of the Bloch oscillations. Following subsequent Bragg reflections, the condensate becomes increasingly diffuse and fragmented [Fig. 4(d)], with clear asymmetry under reflection about the $x$ axis.

\section{CONCLUSION}

We have investigated how the presence of a single soliton or vortex in the initial state of a BEC affects its dynamics in an optical lattice. The initial topological excitation can strongly disrupt the formation of the standing wave on Bragg reflection, thereby creating additional excitations, which cause fragmentation of the atom cloud and damping of the Bloch oscillations.

Our investigation of the dynamics of excited BECs in optical lattices provides further evidence that topological excitations play a key role in the evolution of the atom cloud and in the damping of the center-of-mass motion. Following Bragg reflection, inhomogeneities in the atom cloud can grow rapidly, thereby causing severe damping and a catastrophic breakdown of the transport processes. Our work therefore has implications for understanding the transport and decoherence of condensates in optical lattices. Furthermore, the damping is associated with complex changes in the structure of the atom cloud, such as the formation of skyrmions. Our results suggest that accelerating excited condensates through an optical lattice could provide a route to making and studying skyrmionic excitations, which have previously been inaccessible to experiments on atom clouds. We hope that our work will stimulate further experiments exploring how topological excitations of different types and orientations affect the quantum transport of BECs in optical lattices.
[1] O. Morsch, J. H. Müller, M. Cristiani, D. Ciampini, and E. Arimondo, Phys. Rev. Lett. 87, 140402 (2001).

[2] B. P. Anderson and M. A. Kasevich, Science 282, 1686 (1998).

[3] A. Trombettoni and A. Smerzi, Phys. Rev. Lett. 86, 2353 (2001).

[4] D.-I. Choi and Q. Niu, Phys. Rev. Lett. 82, 2022 (1999).

[5] K. Berg-Sørensen and K. Mølmer, Phys. Rev. A 58, 1480 (1998).

[6] K. P. Marzlin and W. Zhang, Phys. Rev. A 59, 2982 (1999).

[7] M. L. Chiofalo and M. P. Tosi, Phys. Lett. A 268, 406 (2000).

[8] S. Burger, F. S. Cataliotti, C. Fort, F. Minardi, M. Inguscio, M. L. Chiofalo, and M. P. Tosi, Phys. Rev. Lett. 86, 4447 (2001).

[9] V. V. Konotop and M. Salerno, Phys. Rev. A 65, 021602 (2002)

[10] B. Wu and Q. Niu, Phys. Rev. A 64, 061603 (2001).

[11] Z. Dutton, M. Budde, C. Slowe, and L. V. Hau, Science 293, 663 (2001).

[12] S. Burger, L. D. Carr, P. Öhberg, K. Sengstock, and A. Sanpera, Phys. Rev. A 65, 043611 (2002).

[13] S. Burger, K. Bongs, S. Dettmers, W. Ertmer, K. Sengstock, A. Sanpera, G. V. Shlyapnikov, and M. Lewenstein, Phys. Rev. Lett. 83, 5198 (1999).

[14] J. Denschlag et al., Science 287, 97 (2000).

[15] M. Holthaus, J. Opt. B: Quantum Semiclassical Opt. 2, 589 (2000).

[16] M. Cerimele, M. Chiofalo, F. Pistella, S. Succi, and M. Tosi, Phys. Rev. E 62, 1382 (2000).

[17] D.-I. Choi and Q. Niu, Phys. Lett. A 318, 558 (2003).

[18] R. G. Scott, A. M. Martin, T. M. Fromhold, S. Bujkiewicz, F. W. Sheard, and M. Leadbeater, Phys. Rev. Lett. 90, 110404 (2003)

[19] K. E. Strecker, G. B. Partridge, A. G. Truscott, and R. G. Hulet, Nature (London) 417, 150 (2002).

[20] M. R. Matthews, B. P. Anderson, P. C. Halijan, D. S. Hall, C.
E. Wieman, and E. A. Cornell, Phys. Rev. Lett. 83, 2498 (1999).

[21] B. P. Anderson, P. C. Halijan, C. A. Regal, D. L. Feder, L. A. Collins, C. W. Clark, and E. A. Cornell, Phys. Rev. Lett. 86, 2926 (2001).

[22] K. W. Madison, F. Chevy, W. Wohlleben, and J. Dalibard, Phys. Rev. Lett. 84, 806 (2000).

[23] J. R. Abo-Schaeer, C. Raman, J. M. Vogels, and W. Ketterle, Science 292, 476 (2001).

[24] C. Raman, M. Köhl, D. S. Durfee, C. E. Kuklewicz, Z. Hadzibabic, and W. Ketterle, Phys. Rev. Lett. 83, 2502 (1999).

[25] D. L. Feder, M. S. Pindzola, L. A. Collins, B. I. Schneider, and C. W. Clark, Phys. Rev. A 62, 053606 (2000).

[26] B. Jackson, J. F. McCann, and C. S. Adams, Phys. Rev. A 61, 051603 (2000).

[27] J. Ruostekoski and J. R. Anglin, Phys. Rev. Lett. 86, 3934 (2001).

[28] T. Busch and J. R. Anglin, Phys. Rev. Lett. 84, 2298 (2000).

[29] R. G. Scott, A. M. Martin, S. Bujkiewicz, T. M. Fromhold, N. Malossi, O. Morsch, M. Cristiani, and E. Arimondo, Phys. Rev. A 69, 033605 (2004).

[30] M. Abramowitz and I. A. Stegun, Handbook of Mathematical Functions (Dover, New York, 1965).

[31] R. G. Scott, S. Bujkiewicz, T. M. Fromhold, P. B. Wilkinson, and F. W. Sheard, Phys. Rev. A 66, 023407 (2002).

[32] E. A. Burt, R. W. Ghrist, C. J. Myatt, M. J. Holland, E. A. Cornell, and C. E. Wieman, Phys. Rev. Lett. 79, 337 (1997).

[33] W. H. Press, S. A. Teukolsky, W. T. Vetterling, and B. P. Flannery, Numerical Recipes, the Art of Scientific Computing (Cambridge University Press, Cambridge, 1994).

[34] M. L. Chiofalo, S. Succi, and M. P. Tosi, Phys. Rev. E 62, 7438 (2000).

[35] N. W. Ashcroft and N. D. Mermin, Solid State Physics (W. B. Saunders, Fort Worth, 1976). 